\title{
El Enfoque del Derecho al Hábitat en la Educación Formal de Galicia
}

\author{
The Right to Habitat Approach in the Formal Education of \\ Galicia
}

\section{A Abordagem do Direito ao Habitat na Educação Formal da Galicia}

\author{
Xosé Manuel Rosales ${ }^{1 *}$ \\ Soledad Bugallo ${ }^{2}$ \\ Marta Casal ${ }^{2}$ \\ Lucía García-Cernuda ${ }^{2}$ \\ Juan A. Ortiz ${ }^{2}$
}

${ }^{1}$ Colegio de Arquitectos de Galicia. ${ }^{2}$ Arquitectura Sin Fronteras Galicia

\begin{abstract}
El artículo aborda la experiencia de trabajo conjunto dentro del Plan Proxecta entre Arquitectura sen Fronteiras, una Organización No Gubernamental, y el Proxecto Terra, del Colegio Oficial de Arquitectos de Galicia. Se amplía conceptualmente la acción conjunta en los centros educativos a través de la visibilización, reconocimiento, apropiación, transformación y reflexión en torno a las diversas realidades y la vindicación de la "justicia social" mediante el fomento de la acción significativa y participativa del alumnado empleando sus propios saberes y conocimientos. Esta articulación de la mirada socioafectiva significativa y situada forma parte de la transformación real de la realidad del hábitat.
\end{abstract}

Descriptores: Hábitat, Educación, Género, Derechos humanos, Arquitectura.

The article tries to approach the experience of joint work within the Plan Proxecta entre Arquitectura sen Fronteiras, a non-profit organization, and the Proxecto Terra, of the Official College of Architects of Galicia Their jointly line of action in schools is conceptually broadened through visibility, recognition, appropriation, transformation and reflection around the various realities and vindications of "social justice" through the promotion of significant and participative actions of the students using their knowledge. This articulation of the significant and situated socio-affective gaze is part of the real transformation of habitat's reality.

Keywords: Habitat, Education, Gender, Human rights, Architecture.

Este artigo aborda a experiência de um trabalho coletivo no âmbito do Plan Proxecta entre Arquitectura sen Fronteiras, uma ONG, eo Proxecto Terra, o Colégio Oficial de Arquitectos da Galiza. Aborda-se conceptualmente a acção conjunta nos centros educativos através do reconhecimento, apropriação, transformação e reflexão sobre as diversas realidades e a reivindicação da "justiça social” através da acção significativa e participativa dos alunos utilizando os seus saberes e conhecimentos. Esta articulação de uma abordagem sócio-afectiva é parte da transformação concreta da realidade do habitat.

Palavras-chave: Habitat, Educação, Gênero, Direitos humanos, Arquitetura.

*Contacto: proxectoterra@coag.es

ISSN: 2254-3139

www.rinace.net/riejs/

revistas.uam.es/riejs
Recibido:

15 de diciembre 2016

$1^{\text {a }}$ Evaluación: 2 de enero 2017

$2^{\text {a }}$ Evaluación 27 de enero 2017

Aceptado: 29 de enero 2017 


\section{Introducción}

En este artículo se quiere abordar la colaboración del Proxecto Terra (en adelante PT) y Arquitectura Sin Fronteras Galicia (en adelante ASFG) para trabajar en la comunidad educativa de Galicia el derecho al hábitat. Introducir el enfoque de derechos sobre el entorno que nos rodea supone la diferencia y la característica principal de nuestro trabajo educativo y es el que se desarrollará a lo largo de este texto.

Para ello presentaremos un registro de la experiencia de ASFG, dentro de la comunidad educativa, en torno al espacio y al hábitat digno y cómo se incorpora ésta en el trabajo sostenido y de gran experiencia en red del PT. Se esbozan por tanto las acciones desde una perspectiva de pedagogía crítica a través del trabajo colaborativo y transversal. Comenzaremos para ello por aproximar el encuadre de trabajo de las propuestas educativas de carácter innovador que se insertan dentro del denominado Plan Proxecta, en el contexto de la educación formal del ámbito territorial autonómico de Galicia, España. El trabajo conjunto ofrece una óptica ampliada del programa educativo consolidado del PT al que se incorpora el "enfoque de derechos" por parte de ASFG sobre el hábitat.

El Plan Proxecta es una iniciativa institucional del gobierno regional de la Xunta de Galicia mediante la Consellería de Cultura, Educación y Ordenación Universitaria, a través de la cual diversos organismos (acción social, colegios profesionales, tercer sector, etc.) interaccionan con la comunidad educativa de cara a motivar y fomentar la innovación educativa en los centros de educación reglada de la comunidad autónoma. Se desarrolla fundamentándose en las bases competenciales que estructuran parte de los currículos educativos buscando una transversalización de las mismas, así como mediante la implementación de nuevas miradas teniendo como eje fundamental una educación en valores.

\section{Proxecto Terra}

Tras años realizando una labor de innovación, el PT creado por el Colegio de Arquitectos de Galicia en el año 2000, viene desarrollando un proyecto de cooperación con la comunidad educativa a través de un programa completo de contenidos y materiales de cara a proporcionar un soporte para el análisis y comprensión del "Espacio", entendido este como ámbito en el cual el ser humano se relaciona con su medio, donde éste se ve condicionado su perceptividad y construye parte de su cosmovisión existencial.

En este sentido, los objetivos del programa en el ámbito de la educación formal, se centran en las distintas etapas de la Educación Obligatoria -Infantil, Primaria y Secundaria- buscando una mejora de la formación del alumnado y el profesorado de Galicia sobre "las gentes, los espacios y lugares que habitamos" (Proxecto Terra, 2010, p. 1). En esa tarea, se han elaborado materiales específicos que amplían y mejoran de manera transversal los materiales ya incorporados en los currículums escolares legislativos, y se han propuesto actividades complementarias de cara a poder ofrecer alternativas para una comprensión espacial. Las ideas que guiaron esta labor desde los inicios podríamos resumirlas en: universalidad, globalidad, integración curricular, generalidad, permanencia, diversidad, experimentación, interdisciplinariedad, vivencia e interterritorialidad. 
La propuesta se desarrolla segmentada en torno a los ciclos formativos, trabajando en la Educación Primaria tres escalas espacio-temporales, ajustadas a cada uno de los ciclos, $1^{\circ}$ Ciclo: Los espacios inmediatos, $2^{\circ}$ Ciclo: Los espacios intermedios y $3^{\circ}$ Ciclo: Los espacios lejanos, donde el objeto es trabajar sobre el aprendizaje espacial perceptivo. En la Secundaria se proponen cuatro ámbitos de reflexión, la Arquitectura Popular, Arquitectura Contemporánea, la Identidad Territorial y por último los recientes materiales sobre el paisaje en torno al concepto de PAGUS.

Para el desarrollo de esta labor se establecieron tres vías de intervención complementarias: elaboración de unidades didácticas y materiales de apoyo, formación del profesorado y visitas guiadas de arquitectura y ciudad e intercambios territoriales que, año tras año, son ofertadas a profesorado y centros educativos de toda la comunidad autónoma.

\section{Arquitectura sin fronteras}

El trabajo de educación para el desarrollo de ASFG se articula desde el año 2010 alrededor del eje central del "derecho al Hábitat". Este es un derecho cuya garantía implica el cumplimiento de muchos otros derechos en las dimensiones físicas, sociales, políticas y culturales que componen la vida de las personas y comunidades. Es, por lo tanto, un pilar central, una raíz desde la que poder trabajar de manera integral muchos aspectos vinculados a los derechos humanos, y también aquellos transversales y transformadores relacionados con la lucha contra la pobreza, el medioambiente, la interculturalidad, el género o las maneras de gobernanza y de participación social.

Durante los últimos años, ASFG ha desarrollado una intensa labor para impulsar esta línea educativa vertebrada sobre la base del concepto de hábitat que articula de manera holística e integral su defensa a través de un enfoque de derechos. El concepto de hábitat se encuadra dentro de una compleja e imbricada relación entre las esferas de lo físico, lo sociocultural y lo político en las cuales se aglutinan transversalmente la perspectiva de género, transculturalidad, medioambiente y enfoque de derechos. Este concepto se basa en una reconfiguración de las diversas escalas de actuación en torno a la escala humana y la ética de los cuidados. Sobre este supuesto se articulan las diversas acciones que en el plano educativo y formativo se realizan en los centros educativos y colectivos en situación de exclusión social en el área geográfica de Galicia, específicamente en el entorno del área metropolitana de A Coruña y sus hábitats precarios.

\section{Trabajo conjunto}

En el marco de este trabajo y tras exponer la labor que de manera particular ha desarrollado cada una de las entidades, procederemos a tejer diversas líneas y acciones que enmarcan parte de su trabajo conjunto correspondiente a un proceso que comienza en 2014 y se consolida en 2015 dentro del mencionado Plan Proxecta.

La propuesta de acción en los centros educativos nace de la necesidad de introducir en los propios procesos formativos y formadores los valores éticos en torno a la justicia social y la equidad referidos al espacio y el hábitat. Al analizar la ausencia de una visión espacial, como hemos mencionado anteriormente, por una mayor concepción de aspectos relacionados con el Tiempo en los currículos, hace que sea el trabajo sobre el Espacio como base a la estructura analítica y entroncada dentro de una labor conjunta entre entidades y comunidad educativa. A este respecto la red y el soporte de PT es fundamental para su aproximación. 
A través de esta estrategia conjunta e imprescindible se aúna el proceso de difusión de los valores territoriales y de la arquitectura por parte del PT al cual se incorpora el enfoque de ASFG de visibilizar los elementos con los que convivimos en nuestro hábitat, desde el mencionado enfoque de derechos para la defensa de un hábitat digno contando con la mirada implicada de la persona (diversidad de edades y géneros) como generadora del mismo. Para ello, los partimos de una visión de la cultura como un hecho de acción social, que por tanto no ve ésta como un hecho estático sino dinámico (Díaz de Rada, 2010). Se pretende como objetivo general:

Contribuir a mejorar el conocimiento sobre los espacios que habitamos y sobre la identidad territorial de Galicia, mediante la toma de conciencia de la implicación y responsabilidad de la ciudadanía en la construcción y puesta en valor de estos espacios. (Proxecto Terra, 2010, p. 1)

\section{Fundamentación teórica}

\section{1. ¿Qué es derecho al hábitat?}

A nivel teórico nos basamos en la noción de hábitat, como concepto complejo y multidimensional que aglutina diversas dimensiones. Como dicen Giraldo, Bateman y García (2009),

más allá de la ocupación física de un territorio conformado por viviendas y entorno, el hábitat constituye el referente simbólico, histórico y social en el que se localiza el ser humano de manera multidimensional: política, económico-social y estéticoambiental, actuando complejamente en una cultura determinada. (p. 43)

Se concluye por tanto como el lugar, en el cual tanto lo simbólico como lo físico conviven y al cual haremos referencia. Es por ello que partimos de este concepto y de las implicaciones que, como soporte físico, incorpora al ser humano con sus experiencias subjetivas; evitando por tanto trabajar con espacios anónimos sino con aquellos que tienen sentido por ser escenario de sus vivencias propias (Leff, 1998).

Es preciso resaltar la necesidad de introducir el enfoque de derechos. Remarcando entre ellos el Derecho al Hábitat, reconocido en el artículo 25.1 de la Declaración Mundial de los Derechos Humanos (ONU, 1948) así como en el artículo 11 del Pacto Internacional de Derechos Económicos Sociales y Culturales (ONU, 1966). E incluir además la propuesta incluida dentro de la Declaración Universal de los Derechos Humanos Emergentes (Institut Drets Humans Catalunya, 2009). Por su parte, el Derecho a la Ciudad se entiende como derecho de la ciudadanía a vivir en territorios apropiados para la convivencia y ricos en usos diversos, donde el espacio y los equipamientos públicos son factores de desarrollo colectivo. Esta temática es también eje de debate constante en las últimas ediciones del Foro Urbano Mundial y del Foro Social Mundial, y cabe destacar por ello el día Mundial del Hábitat. Resaltamos a este respecto la celebración del Hábitat III, en septiembre de 2016, donde se aglutina el debate en torno a varias de las problemáticas globales que se plantean de cara a promover un hábitat digno y sostenible, en la cual se ha pretendido incorporar una revisión sobre los Objetivos de Desarrollo Sostenible, aprobados en 2015, tras una revisión del fracaso de los Objetivos de Desarrollo del Milenio fijando nuevos objetivos globales para el años 2030.

Consideramos fundamentales, entre otros, dos aportes teóricos y de enfoque sobre la construcción y definiciones de los derechos humanos y de las necesidades humanas, a la hora de trabajar con el alumnado el derecho al hábitat. 
En el apartado teórico de los derechos internacionales cabe realizar una serie de matizaciones, como las realizadas por Boaventura de Sousa (2010) con respecto a la reconstrucción intercultural de los derechos humanos y su paso a una posible descolonización del saber basado en su propuesta de la ecología de los saberes. Además de esta puntualización, cabe resaltar la revisión desde el ámbito de la consideración horizontal de las necesidades y la incorporación de la noción de desarrollo a escala humana (Max-Neff, Elizalde y Hopenhayn, 2010) de cara a motivar una consideración de las propias necesidades y las relaciones sistémicas entre diversas geografías y, en nuestro caso, de las necesidades del alumnado como sujetos activos de ciudadanía real.

Se concentra y sustenta en la satisfacción de las necesidades humanas fundamentales, en la generación de niveles crecientes de autodependencia y en la articulación orgánica de los seres humanos con la naturaleza y la tecnología, de los procesos globales con los comportamientos locales, de lo personal con lo social, de la planificación con la autonomía y de la sociedad civil con el Estado (Max-Neff, Elizalde y Hopenhayn, 2010).

El derecho al hábitat estructura, por tanto, el concepto del análisis de las relaciones existentes a nivel espacial y relacional a través del enfoque de derechos y vinculado con los derechos de la infancia (UNICEF, 2006) donde se articula la necesidad de trabajar este concepto en el ámbito educativo. Es básico para entender y trabajar en las causas estructurales de las desigualdades. Considerar abordarlo en el ámbito de la educación se hace, por tanto, perentorio para ASFG, posicionada desde la realidad de agente complementario a la práctica del profesorado. A este respecto, se hace preciso exponer el encuadre de la educación, entendida inicialmente para el desarrollo, hasta la actual perspectiva de la Educación para la Ciudadanía Global que se basa, como resaltan Mesa y Escudero (2011), en una ciudadanía crítica en sintonía con gran parte de los presupuestos teóricos que Paulo Freire inicia con su Pedagogía del Oprimido. De esta forma el trabajo de la temática del hábitat se integra en la propuesta educativa de ASFG y pasa a incorporarse a la colaboración con PT.

A este respecto, el trabajo complementario entre PT y ASFG considera la mediación entre lo objetual y lo intersubjetivo de las relaciones entre las dimensiones físicas, políticas y económicas (De Manuel, 2010) que introducen una mirada integrada del alumnado como sujeto activo y necesario y donde el profesorado actúa como mediador en los procesos de análisis y comprensión crítica del Espacio y, en particular, del hábitat. Se precisa reconocer las necesidades propias del alumnado y tratar de motivar su visión crítica sobre la realidad a través de valores éticos de justicia social de cara a la búsqueda de la autonomía propia (Freire, 2011).

\subsection{Aspectos transversales incorporados}

Las líneas de trabajo se centran en la perspectiva de género, inter/transculturalidad y medioambiental. Estas son líneas habituales dentro de la línea de la Educación para el Desarrollo.

Las diversas acciones se desarrollan dentro de un enfoque pedagógico abierto y creativo, empleando metodologías enfocadas a la adquisición no sólo de conocimientos, sino también de metodologías práctico-teóricas (Freire, 2009), capacidades socio-afectivas, y al impulso de procesos de facilitación de la autonomía personal y colectiva. Para ello, se procede a incorporar directamente en las propuestas los siguientes aspectos: 
- Adquisición de conocimientos sobre el hábitat desde un enfoque integral, social y de derechos que permita generar una visión crítica, compleja e interrelacionada del mundo que vivimos.

- La dimensión de género se incluye en todas las actuaciones; tanto en las acciones desarrolladas como en los contenidos, como eje vertebrador de las transformaciones de los roles en los cuales se socializa el alumnado, así como el análisis de la constatada feminización de la pobreza y las diversas violenciasconflictos que las estructuras patriarcales generan entre los diversos géneros.

- La perspectiva trans-inter-cultural, de manera crítica, y visualizando las opresiones y relaciones de poder que por razón de pertenencia étnico-cultural impregnan las relaciones en el mundo concreto y próximo, así como también en los contextos geográficos y culturales diferentes desde una óptica poscolonial.

- Introducción de conocimientos teórico-prácticos sobre la visión sobre las maneras de gobernanza respeto del hábitat. Buscando un aprendizaje colaborativo y mediante la participación activa del alumnado (Hart, 1993) con sus opiniones propias, valoraciones situadas desde su propia realidad. Indagando por tanto en las posibilidades de su papel activo y necesario en la construcción del mismo, como ya Francesco Tonucci (1993) relaciona a la ciudad y la infancia.

- Adquisición de capacidades personales y colectivas, a nivel cognitivo y socioafectivo para desarrollar de manera satisfactoria procesos colectivos, en un ejercicio de ciudadanía crítica organizada por un objetivo común. Posibilitando la incorporación paulatina de la participación (Apud, 2010) como elementos de transformación posible y consciente. Además, aprender sobre los modelo de gobernanza, posibilita la experimentación de forma vivencial y lúdica un proceso de tendente a la metodología de la investigación-acción-participativa (IAP) (Eizaguirre y Zabala, s.f.) vivenciando las ventajas del trabajo colaborativo e identificando los puntos problemáticos disteis procesos, aprendiendo a gestionar las emociones y los conflictos que surgen.

- Asimismo se considera fundamental el enfoque transdisciplinar. El intercambio de impresiones dentro del ámbito educativo, así como con profesionales con presencia o experiencia fuera del ámbito local para mostrar y contextualizar las problemáticas y las maneras diversas de resolverlas. Mostrar diversas maneras de afrontar las problemáticas vinculadas al hábitat, que afecta la toda la población mundial a diversas escalas y que alcanza sin embargo a la toda la ciudadanía, pero situándose y huyendo de una visión única y homogeneizadora de la realidad (Boaventura de Sousa, 2010).

En definitiva plantamos metodologías y enfoques pedagógicos constructivistas, de motivación de una reflexión autónoma crítica y no sesgada, con la creación de conocimiento por parte de los propios destinatarios de la formación. Con un aprendizaje profundamente situado (realidad inmediata y concreta de un continuum rururbano) y poniéndola en relación con otras realidades, ayudando a tomar conciencias de las dinámicas de poder que cristalizan en el hábitat y la toma de conciencia sobre los privilegios y opresiones que sufrimos, favoreciendo la empatía con otras personas y colectivos, y motivando en última instancia a un cambio personal y colectivo hacia transformación social. 


\section{Método}

Los métodos desarrollados por ASFG en el ámbito educativo son diversos, si bien todos ellos pretenden contribuir a trabajar en todas o algunas de las fases señaladas en la ilustración anterior. Concretamente, en nuestro trabajo de colaboración con PT focalizamos el trabajo en las primeras fases del proceso que se presenta. Principalmente nos centramos en aprender a "ver”, “analizar”, "reflexionar” y "soñar” sobre distintos aspectos que afectan al hábitat del alumnado (figura 1).

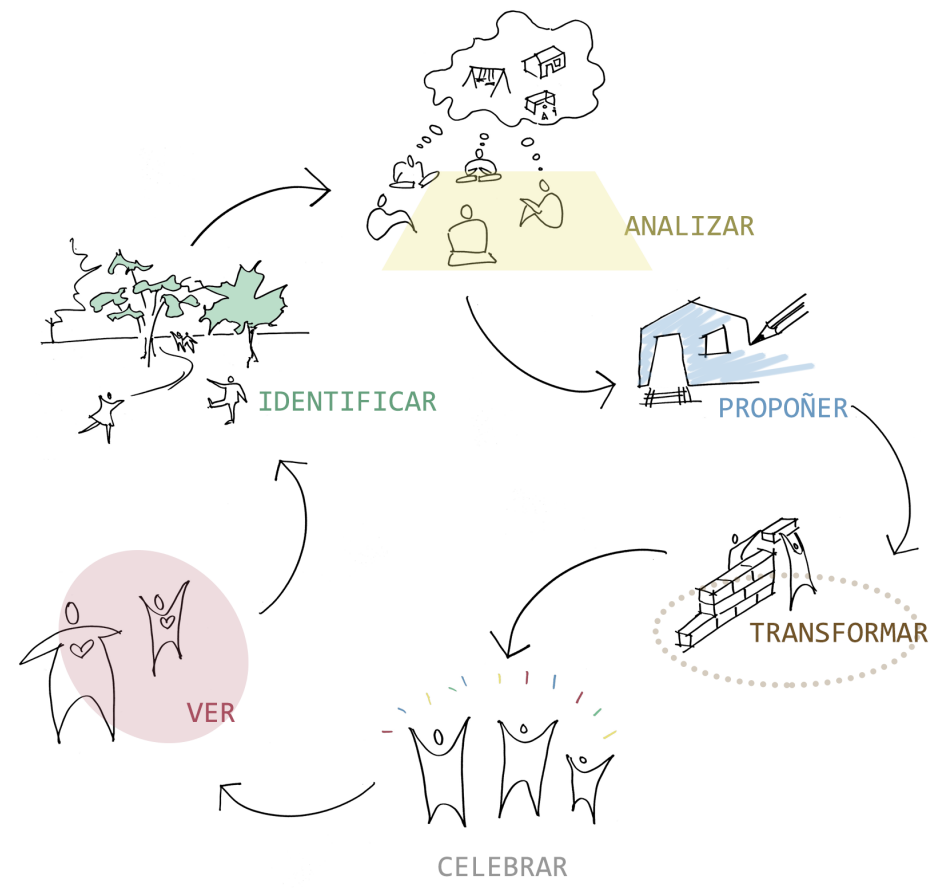

Figura 1. Fases que se abordan en el trabajo del alumnado Fuente: Elaboración propia.

A través de diversas metodologías y propuestas críticas, vivenciales, constructivistas y participativas queremos contribuir a que el alumnado con el que trabajamos aprenda a ver y mirar su entorno desde otros prismas que les permitan desarrollar la capacidad de analizarlo críticamente, poniendo en valor aquello que aporta a una vida digna $\mathrm{y}$ proponiendo alternativas a aquellos aspectos que hacen su hábitat, y sus vidas, precarias.

\subsection{Principios metodológicos básicos}

\section{a) Aprendizaje significativo y socioafectivo}

En el cual la interacción de los conocimientos del alumnado sean puestos de relieve como eje vertebrador del propio aprendizaje situado. Articulando por tanto la acción real con el aprendizaje activo, directamente relacionado con el alumnado permitiendo un trabajo hacía la capacidad crítica y propositiva. En este sentido incorporando tanto las emociones, como ideas y propuestas.

\section{b) Aprendizaje cooperativo y participativo}

En el cual el encuentro inter-subjetivo sea hecho relacional entre personas de forma equitativa, incorporando las diversas formas de pensar, motivando el diálogo, debate, la 
escucha activa, el contraste, y la argumentación. Es por ello que se hace necesaria su puesta en práctica en ambiente de confianza y respeto.

c) Análisis crítico, reflexión y evaluación

Entendida a nivel procesual durante todo el desarrollo de las acciones, de cara a su ajuste continuo, identificando errores y problemáticas que dificultan el poder desarrollar las actividades propuestas, para ello posibilitado el intercambio de pareceres, así como de registro de las intervenciones de cara a analizar resultados y procesos de trabajo en base a las premisas de partida.

Pasamos ahora a analizar diversas herramientas empleadas desde ASFG para el desarrollo del trabajo en las aulas, de las cuales destacamos específicamente tres: la Unidad didáctica derecho al hábitat, Diagnóstico participativo y Recursos audiovisuales complementarios.

\subsection{Unidad didáctica: El derecho al hábitat}

Como complemento al material desarrollado por el PT, la guía del profesorado sobre el derecho al hábitat pretende ampliar el marco teórico e implementar el concepto desde el enfoque de derechos, así como la realización de diversas dinámicas ajustadas entorno al mismo concepto y acercando realidades de hábitats precarios en diversos contextos que complementan la propuesta existente.

En el año 2013, elaboramos una unidad didáctica sobre Derecho al Hábitat contando para ello con la cooperativa "Hábitat social". Esta unidad didáctica surgió de la necesidad de contar con un material de base para trabajar con alumnado de secundaria principalmente, y que pudiese ser empleado directamente por el profesorado para poder llegar de esta forma a mayor cantidad de personas y lograr más impacto.

El objetivo general del material era "motivar la reflexión del alumnado entorno al hábitat como un sistema complejo de situaciones que debe construirse colectivamente y al que todas las personas tienen derecho" (Hábitat Social, 2013, p. 7). Los objetivos específico desarrollados en la guía son los siguientes:

- Distinguir los conceptos de hábitat y hábitat humano.

- Identificar y reconocer situaciones representativas en relación al hábitat más próximo de cada alumno/a.

- Familiarizarse con las herramientas digitales para la creación de una cartografía.

- Analizar las situaciones escogidas por cada alumno/a e interpretar los aspectos positivos y negativos de cada una de ellas.

- Relacionar los elementos comunes en las diferentes situaciones del hábitat de cada alumno/a.

- Identificar las características de un hábitat adecuado.

- Asimilar el concepto de complejidad del hábitat y del derecho al hábitat a través de las situaciones analizadas.

- Reconocer el concepto de construcción colectiva. 
Para conseguir los objetivos marcados, se propone una estrategia metodológica con dos características principales:

- La componente práctica prima sobre la teórica, procurando la participación activa del alumnado de forma que se consiga su atención e interés.

- La realidad objeto de reflexión es la próxima, la que el alumnado puede reconocer e identificarse con ella, fomentando un aprendizaje significativo.

Con esta perspectiva, el núcleo de la unidad didáctica queda constituido por un trabajo de campo a realizar por el alumnado, y por un tiempo de exposición y debate posterior en el que el material recogido sirve de apoyo a la reflexión. Esta secuencia implica un desarrollo temporal de la unidad en dos módulos o clases, que además deberán estar separados en el tiempo para dar cabida al trabajo fuera del aula.

El primero de los módulos comprenderá básicamente la contextualización de la unidad, en la que el profesorado presenta de manera breve el tema a tratar, para el cual dispone de un material gráfico de apoyo elaborado específicamente para esta propuesta. Además, se propone al alumnado la propuesta del ejercicio a realizar fuera del aula y la introducción de la herramienta digital que se empleará para éste: la aplicación de cartografía digital Crowdmap.

El trabajo de campo se llevará a cabo de manera individual para incidir en la relación entre el hábitat y la experiencia personal del alumno/a, en la búsqueda de percepciones e identificaciones particulares que retratará a través de fotografías. Estas fotografías son subidas y geolocalizadas por el alumnado en la aplicación Crowdmap. En esencia, el resultado será una cartografía colaborativa de la relación sensible entre el hábitat y ese grupo de alumnos y alumnas, que además se podrá superponer a la de otros grupos del mismo centro o de otro centro educativo.

El segundo módulo trata de poner en común y justificar el material realizado así como abrir un espacio de debate en el que se puedan interrelacionar ideas e intercambiar opiniones entorno a lo concreto, con la cartografía elaborada como soporte para conocer las diferentes visiones del alumnado, según sea el contexto que habita.

Puesto que la lógica de esta unidad didáctica es la de tener un breve acercamiento a los conceptos relacionados con el hábitat, no existirá una evaluación específica de los conocimientos adquiridos por el alumnado sino que, en todo caso, se observarán sus actitudes y motivaciones de cara a extraer una conclusión sobre el interés de la misma.

Esta unidad didáctica fue puesta en práctica por parte del voluntariado de ASFG en diversos centros educativos de Galicia durante el año 2016, en el marco de la relación de colaboración con el PT, llegando a centros educativos de ámbito rural y urbano. Esta experiencia piloto ha puesto de manifiesto la necesidad de seguir profundizando en esta colaboración ya que ha sido una práctica educativa bien valorada tanto por el profesorado como por el alumnado.

Cabe destacar que en estas experiencias hemos ido adaptando la metodología a los núcleos temáticos de interés que cada centro venía trabajando en su propio proceso, coordinándonos previamente con el profesorado para conocer su trabajo en este ámbito en el marco del PT y poder aportar en aquellos aspectos más significativos o necesarios para ellos. 
La adaptación de la propuesta se realizó también para poder trabajar con edades más tempranas, llegando a aplicarla también con alumnado de primaria. En estos casos por ejemplo, hemos adaptado la propia metodología, rescatando los aspectos esenciales de la misma en cuanto a los conceptos básicos a trabajar, los espacios de reflexiones individuales y colectivas respecto al hábitat, pero introduciendo para el análisis dibujos elaborados por el alumnado, en lugar de fotografías y el Crowdmap.

\subsection{Un proceso de diagnóstico participativo como herramienta de educativa}

En el año 2015 realizamos un pequeño diagnóstico participativo con alumnado de $2^{\circ}$ y $3^{\circ}$ de la ESO en tres centros educativos del ámbito de la ciudad de A Coruña, con la colaboración de la cooperativa Feitoría Verde y Miguel Pardiellas. Participaron casi 200 alumnos y alumnas, así como sus familias en algunas fases del proceso.

El objetivo principal de este proceso era profundizar en el conocimiento y percepción que el alumnado tenía de su hábitat inmediato, así como de lo que percibía como un hábitat digno o un hábitat precario. Queríamos recoger información cuantitativa y cualitativa sobre conocimientos, percepciones, necesidades y aptitudes del alumnado y sus familias que nos ayudasen a validar el enfoque y contenidos de nuestras actividades educativas y a replantear o profundizar en aquellos aspectos que fuese necesario.

Para ello el diagnóstico desarrolló varias técnicas de recogida de información. Por una parte un cuestionario que fue cubierto por el alumnado y por sus familias de forma independiente. En el mismo indagamos en aspectos relacionados con:

- Satisfacción con el hábitat en el que viven.

- Percepción y modos en los que el hábitat afecta a su vida cotidiana.

- Percepción sobre la posibilidad de incidencia y transformación del hábitat en el que viven.

- Identificación de elementos que conforman un hábitat digno y un hábitat precario.

- Impacto de la pertenencia cultural o el género en el hábitat y en la percepción del mismo.

- Importancia de la participación ciudadana en la gestión del hábitat.

- Percepción sobre la movilidad en relación al transporte privado.

También pedimos a los niños y a sus familias que realizasen un dibujo sobre su hábitat. En estos dibujos observamos varias cosas significativas que nos dan pautas para enfocar nuestro trabajo educativo para trabajar desde una perspectiva crítica la construcción social del hábitat:

- Inexistencia de la figura no $t$ en una ciudad con mar y zonas verdes como A Coruña.

- Dibujo esquemático y descriptivo de un contexto urbano frente a un dibujo más interpretativo o imaginativo.

- Escasez de elementos paisajísticos y de espacios de ocio no vinculado al consumo. 
- Movilidad representada de forma individual a través de la figura central del coche. No aparecen medios públicos de transporte y solo dos bicicletas en todos los dibujos elaborados.

Una de las acciones de mayor impacto tanto para el proceso como para el propio alumnado participante fueron las Derivas. Las derivas consistieron en salidas organizadas del alumnado para analizar su entorno inmediato en relación a unos ejes temáticos dados. El alumnado se dividió en grupos de 5 o 6 personas que tenían que realizar paseos, derivas, entorno a su centro educativo moviéndose libremente en un área determinada previamente sobre un plano. En esos paseos a cada grupo se le daba un tema de observación en relación a aspectos significativos que fueron detectados en los cuestionarios previos.

Los temas escogidos fueron: movilidad, cultural, género, ocio, mal barrio/buen barrio.

Se les asignó un eje temático de observación a cada grupo y éste debía pasear durante una hora, a la deriva, e ir fotografiando aquello que relacionaran de alguna forma con el tema de observación. El alumnado no tenía que llegar a acuerdos sobre qué fotografiar, sino que debían de recoger lo que fuese significativo para cada persona, pudiendo debatir y discutir sobre el tema. Cada grupo iba con una persona como acompañante, a cierta distancia, con el objetivo de observar el proceso, los debates que se generaban, qué observaban y qué no, y solucionar dudas puntuales sobre la metodología. Una vez terminado el recorrido el alumnado los diferentes grupos de observación compartieron en plenaria la experiencia y las observaciones realizadas, así como los debates generados durante la deriva.

Los resultados de las derivas y el proceso mismo, aportaron mucha y relevante información para identificar la diversidad en la percepción del alumnado sobre los temas planteados. Los tópicos en relación a estos aspectos que es necesario trabajar, así como aquellos sobre los que tenían una visión crítica más elaborada. Las dificultades de identificar elementos del hábitat con los temas planteados.

Este proceso se cerró con un taller de dos horas de duración orientado a reflexionar sobre el impacto de hábitat en nuestras vidas. Sobre como este nos afecta de forma diferente según nuestra edad, sexo, procedencia, clase social o diversidad funcional. Reflexionamos a través de dinámicas de juegos de roles sobre la importancia de una construcción de nuestros entornos desde una perspectiva inclusiva que tenga en cuenta las diversidades y deje de tener como referencia en varón, blanco de clase media y con capacidades típicas". Reflexionamos y vivenciamos como el diseño y gestión de nuestro hábitat puede contribuir a reducir o incrementar desigualdades en el acceso a derechos y recursos y en definitiva a una vida digna. El alumnado tenía además que plantear propuestas para solucionar los diferentes obstáculos que la configuración actual de su hábitat inmediato tenía en la vida de muchas personas y colectivos.

Este proceso de diagnóstico, se concibió inicialmente para obtener información que nos ayudase a evaluar y replantear nuestras propuestas educativas, sin embargo se convirtió en sí mismo en una actividad educativa de alto impacto y con una valoración positiva por parte del alumnado y del profesorado que destacaron los siguientes aspectos:

- Trabajar durante un proceso de varias sesiones el tema del Hábitat desde un enfoque integral (físico, social, político, cultural, simbólico) y de derechos. El 
trabajo sobre el hábitat se aborda habitualmente desde una perspectiva física o ambiental y de forma muy puntual en algunas materias.

- El valor de la experiencia vivencial, grupal y participativa. Las metodologías utilizadas contribuyeron a una mayor implicación del alumnado y del profesorado.

- Enfoque crítico, reflexivo y orientado a la acción sobre temas que nos afectan de forma cotidiana.

- Identificación de problemas por parte del alumnado desde sus perspectivas, experiencias y vivencias situadas, así como la elaboración de propuestas transformadoras haciendo énfasis en las dimensiones de la democracia participativa, de la organización y participación ciudadana y de nuestra capacidad y responsabilidad de incidencia y transformación social.

\subsection{Videoforum: Una brecha en los muros del hábitat precario y de la marginación}

Los videoforum son una herramienta de sensibilización y educación empleada para trabajar principalmente conceptos como: hábitat precario, hábitat digno, igualdad de oportunidades, exclusión social, hábitat e interculturalidad o hábitat y género.

Trabajamos a través de dos cortos documentales que fueron elaborados en A Coruña, ámbito de trabajo del grupo de cooperación local de ASFG, y con la colaboración de Xaime López-Chispón. Describen las condiciones de vida de la población que vive en un asentamiento chabolista de la ciudad, A Pasaxe. Esta población es diversa, pero con un elevado porcentaje de población gitana. Los cortos fueron elaborados contando con la implicación de las personas protagonistas, residentes del asentamiento, que fueron partícipes de todas las fases del proceso. Se concibió como trilogía -diversas edades infancia, personas adultas jóvenes, y personas adultas mayores- mediante un proceso de video participativo en el que la población residente en un hábitat precario, marcado por la exclusión social, fuese sujeto activo del proceso y del mensaje que se quería hacer llegar al resto de la población.

Resaltamos en el trabajo del aula dos de los documentales:

- Pedra, papel, aborda la situación y vivencias de la infancia que reside en el asentamiento. La precariedad de sus viviendas y el entorno en el que viven, el aislamiento físico y social, la dificultad de acceso a derechos, recursos e infraestructuras de la ciudad, etc. (Arquitectura Sin Fronteras y López, 2013).

- A Pasaxe, aborda diversas dimensiones de la vida de dos mujeres jóvenes que crían a sus hijos e hijas solas. Habla de sus dificultades para generar ingresos estables y suficientes, de sus condiciones de vida, sus dificultades y su lucha para satisfacer sus necesidades, sueños y aspiraciones (Arquitectura Sin Fronteras y López, 2014).

Los documentales se proyectan en centros educativos y habitualmente se trabaja con alumnado de la ESO. Nos permiten acercar una realidad muy próxima físicamente al alumnado de A Coruña, pero muy lejana en cuanto al conocimiento y contacto que tienen con la misma, cargado de prejuicios y estereotipos asociados a las condiciones de exclusión social y particularmente a la población gitana. 
La metodología de los videoforum parte de una primera fase de construcción, por parte del alumnado, de la definición de lo que constituye para ellos un hábitat digno, como aquel que nos permite desarrollarnos como personas en todo nuestro potencial. A través de una lluvia de ideas el alumnado va "construyendo" ese hábitat digno con los elementos que considera fundamentales. Inicialmente suelen comenzar por aspectos materiales, ligados a la vivienda, artilugios cotidianos, para pasar posteriormente a una dimensión más allá de su entorno inmediato a otro más social. Identifican así los recursos e infraestructuras públicas que nos permiten acceder a derechos como la salud, la educación, espacios públicos y de ocio, etc. Para finalmente completar esa "construcción" con elementos más vinculados a las relaciones familiares, vecinales, de amistad, en definitiva a los vínculos humanos, los afectos y a nuestra integración social.

Con esta imagen de fondo construida por el alumnado pasamos a ver los documentales para después iniciar un proceso de debate y reflexión sobre los mismos en relación a los aspectos mencionados anteriormente: hábitat precario, igualdad de oportunidades, exclusión social, discriminación y género.

Los documentales contados en primera persona son el primer acercamiento que los niños y niñas tienen hacia esta realidad. La primera impresión es la de que las personas que viven en esos asentamientos se "humanizan". Adquieren rostros concretos, voces, vivencias y aspiraciones. Pasan de estar representados en el imaginario del alumnado por un colectivo homogéneo y estigmatizado a ser personas concretas que se dirigen a los niños y niñas.

En el debate posterior trabajamos estos prejuicios y estereotipos vinculados a esta población que tienen que ver principalmente con visiones fatalistas de su existencia: "no hay otras vidas posibles para ellos", así como a visiones que los responsabilizan en exclusiva de la situación que viven y casi naturalizan y esencializan la relación entre población gitana, hábitat precario y exclusión social. Estos prejuicios están fuertemente arraigados, ya que incluso tras el visionado de los documentales hay muchas resistencias a transitar hacia visiones más críticas de estas situaciones vinculándolas a dinámicas de desigualdad y exclusión social, así como a "mirarnos" y considerar la responsabilidad de la sociedad respecto a estas situaciones.

A través de juegos de dinámicas de roles en los que se implican representamos distintas dinámicas de opresión y situaciones de discriminación que operan cotidianamente en la vida de estas personas. De esta forma conseguimos de una forma más vivencial aproximarnos a las dificultades de romper el círculo de la exclusión y de la necesidad de que todos los actores sociales se movilicen hacia dinámicas de integración vs. asimilación.

El eje transversal de análisis de estas circunstancias es el derecho al hábitat. Así analizamos las relaciones entre hábitat y pertenencia étnica, género o edad. Reflexionamos sobre cómo estas variables pueden facilitar o dificultar el acceso a un hábitat digno y con ello los derechos asociados al mismo. Reflexionamos también sobre la negación del acceso a derechos y recursos en igualdad de oportunidades por el hecho de nacer en contextos precarios y marginalizados, y del impacto que puede tener eso en la vida de la población residente en estos asentamientos, particularmente de los niños y niñas. 
Consideramos que esta herramienta es eficaz para abordar tanto prejuicios y estereotipos vinculados a la población gitana o en situación de exclusión, como para dimensionar la importancia del hábitat, desde un punto de vista integral, en el desarrollo de nuestras vidas y de nuestro potencial como personas. El impacto de esta estrategia de sensibilización y educación tienen mucho que ver con el contenido de los documentales y con el hecho de que desatan una conexión emocional del alumnado con las personas protagonistas, facilitando la empatía y con ello la posibilidad de generar curiosidad, compresión y derribar prejuicios que los deshumanizan y responsabilizan de la situación que viven, y que no hacen más que fortalecer las dinámicas de exclusión y discriminación, y la distancia, física, social y emocional respecto a la realidad que viven millones de personas en el mundo.

\section{Discusión y conclusiones}

El PT ha demostrado su aporte en el ámbito educativo para empoderar al profesorado en el abordaje de temas relacionados con el territorio, el patrimonio y el desarrollo sostenible. Con la elaboración de diversos materiales didácticos adaptados a los diferentes niveles educativos, así como con formaciones específicas para el profesorado ha logrado integrarse en la experiencia educativa de múltiples centros de la comunidad gallega en los últimos años promoviendo proyectos interdisciplinares, dinámicos y contextualizados.

El empoderamiento del profesorado redunda directamente en el alumnado que tiene la posibilidad de adquirir conocimientos sobre su entorno en relación a la arquitectura popular, contemporánea o la identidad territorial. Contribuye así, a mejorar el conocimiento sobre los espacios que habitamos y sobre la identidad territorial de Galicia, mediante la toma de conciencia de la implicación y responsabilidad de la ciudadanía en la construcción y puesta en valor de estos espacios.

El propósito de PT, su filosofía y propuesta metodológica sintoniza con la propuesta que desde ASFG se tiene en relación al Derecho al Hábitat por lo que la colaboración que en este artículo se ha presentado, consideramos que tiene un efecto sinérgico positivo al incorporar la perspectiva de derechos al abordaje del hábitat, así como otras perspectivas interrelacionadas de forma transversal como la perspectiva de género, intercultural o la medioambiental. Esta sinergia vislumbra la idoneidad de tender hacia un trabajo en red, buscando espacios de encuentro entre las propuestas que se realizan en la educación formal. Además de ser positiva la estrategia de dar soporte de herramientas-materiales al profesorado con un enfoque holístico de las temáticas a tratar. Se fomenta la facilidad de encaje en los currículos formales o como alternativa para varias áreas temáticas.

Las dificultades detectadas son en parte motivadas por los tiempos de la educación formal al uso, para propuestas realmente transformadoras. Constatamos la potencia metodológica de la propuesta educativa al implicar de forma directa y vivencial al alumnado. Al relacionar las actividades propuestas con su entorno inmediato, rescatando y poniendo en valor aquello que contribuye a una vida digna, trabajando paralelamente este concepto, así como cuestionando modelos de desarrollo economicistas hegemónicos que no tienen en el centro a las personas y el territorio. Ayudan a encuadrarlo en su realidad próxima y a motivar la capacidad dialógica del alumnado, que conocedor de su entorno perceptivo tiene elaborada una idea propia del mismo, además de facilitar 
colocarse, en su propia aula, en la diversidad de formas de vivir que existen. Esto se ha constatado en la flexibilidad que ofrece la guía didáctica de situar el debate en lugares concretos, y posibilitando una multiplicidad de ópticas donde las problemáticas locales eran enmarcadas por el alumnado, motivando una incipiente visión crítica y propositiva que se considera de interés para poder implementar y dar seguimiento.

Las metodologías constructivistas y colaborativas motivan al alumnado y desatan la fuerza de las experiencias socioafectivas y del aprendizaje significativo, lo que viene a demostrar la importancia de las mismas, así como del papel que el profesorado como mediador de crear estas experiencias, aspecto que el propio proceso de integración en el Plan Proxecta motiva, ya que son los centros quienes solicitan la temática a trabajar.

Por su parte el profesorado tiene la posibilidad de desarrollar propuestas y aprendizajes interdisciplinares, contando con una propuesta articulada para ello y con materiales elaborados que puede adaptar a su contexto específico. Además la colaboración presencial en el centro con actividades educativas de ASFG supone un aporte y apoyo que motiva a seguir en la línea emprendida con el PT, al poder contrastar ideas, propuestas e integrando nuevas herramientas y metodologías para trabajar específicamente el hábitat desde un enfoque de derechos.

Estas propuestas son bien valoradas ya que en el currículum son pocos los contenidos dedicados a los temas abordados en el marco del PT, tratados en la mayoría de los casos de forma parcial, puntual y no contextualizada. Y lo que es más relevante es la carencia de un enfoque de derechos transversalizada en el currículum, algo que proporciona de mayor potencia el enfoque formativo, ya que se plantean cuestiones no solo necesarias sino exigibles por parte de la ciudadanía.

La trayectoria en el tiempo de PT ha derivado en muchos centros educativos en una dinámica propia que ha traspasado las propuestas iniciales, llevando al alumnado no solo a ver, y reflexionar sobre su entorno de forma crítica sino a soñar y realizar pequeñas acciones de transformación en su entorno, estimulando la conciencia de una ciudadanía no sólo crítica, sino con herramientas para la acción y transformación social. Desde ASFG la pequeña aportación a este proyecto como apoyo-facilitador tanto al profesorado como al alumnado para trabajar en este sentido, pasando del ver y analizar a la generación de propuestas/sueños para transformar y celebrar. En este sentido se hace necesario un trabajo sostenido en el tiempo para poder dar seguimiento a procesos completos de intención transformadora.

Trabajar el hábitat desde un punto de vista social, integral y de derechos tiene un gran potencial de cara a descubrir y favorecer el pensamiento crítico de una "ciudadanía" consciente, implicada con su realidad inmediata y con otras más lejanas, identificando situaciones de violación de derechos que pueden ser próximas, contextualizadas y comprometiéndose en el abordaje y creación de alternativas.

A este respecto el introducir las bases de una Educación para la Ciudadanía Global (Mesa y Escudero, 2011), o glocal en su vertiente actual permite, a priori, la reformulación de las diversas propuestas de educación transformadora, en la cual se sobrepasa la visión desde una ONG de acción voluntaria, sino que intentando aglutinar las posibilidades de un trabajo cooperativo y participado activamente, y para el cual la aportación inherente del PT es básica, transformando los mecanismos previos de ASFG de cara a la labor en parte de los centros escolares formales. 


\section{Referencias}

Arquitectura Sin Fronteras. (Prod.) y López, X. (Dir.). (2013). Pedra, papel [DVD]. Madrid: Arquitectura Sin Fronteras.

Arquitectura Sin Fronteras. (Prod.) y López, X. (Dir.). (2014). A Pasaxe [DVD]. Madrid: Arquitectura Sin Fronteras.

Apud, A. (2010). Participación infantil. Madrid: Unicef.

Boaventura de Sousa, S. (2010). Descolonizar el saber. Montevideo: Ediciones Trilce.

Díaz de Rada, A. (2010). Cultura, antropología y otras tonterías. Madrid: Trotta.

De Manuel, E. (2010). Construyendo triángulos para la gestión social del hábitat. Hábitat y Sociedad, 1, 13-37.

Eizaguirre, M. y Zabala, N. (s.f.). Diccionario de acción humanitaria y cooperación al desarrollo. Investigación-acción participativa. Bilbao: Hegoa.

Hábitat Social. (2013). O dereito ó hábitat. Guía do Profesorado. A Coruña: Arquitectura Sen Fronteiras.

Hart, R. (1993). Ensayos Innocenti $N^{\circ} 4$. La participación de los niños. De la participación simbólica a la participación auténtica. Florencia: Unicef.

Freire, P. (2009). Pedagogía de la autonomía. Saberes necesarios para la práctica educativa. Ciudad de México: Siglo XXI.

Freire, P. (201 1). Política y educación. Ciudad de México: Siglo XXI.

Giraldo, F., Bateman, A. y García, J. (2009). Urbanización para el desarrollo humano. Políticas para un mundo de ciudades. Bogotá: ONU-HABITAT

Institut Drets Humans Catalunya. (2009). Declaración universal de derechos emergente. Barcelona: Institut de Drets Humans de Catalunya.

Leff, E. (1998). Saber ambiental: Sustentabilidad, racionalidad, complejidad, poder. Ciudad de México: Siglo XXI.

Max-Neff, M., Elizalde, A. y Hopenhayn, M. (2010). Desarrollo a escala humana. Opciones para el futuro. Madrid: Biblioteca $\mathrm{CF}+\mathrm{S}$.

Mesa, M. y Escudero, J. (2011). Diagnóstico de la educación para el desarrollo en España. Madrid: CEIPAZ - Fundación Cultura de Paz.

ONU. (1948). Declaración de derechos humanos. Recuperado de http://www.ohchr.org/EN/UDHR/Documents/UDHR_Translations/spn.pdf

ONU. (1966). Pacto internacional de derechos económicos sociales y culturales. Recuperado de http://www.ohchr.org/SP/ProfessionalInterest/Pages/CESCR.aspx

UNICEF. (2006). Declaración sobre los derechos del niño. Madrid: UNICEF Comité Español.

Proxecto Terra. (2010). Memoria proxecto terra. Un país: su arquitectura y su territorio. Recuperado de http://proxectoterra.coag.es/wp-content/uploads/2015/08/Web15-01-Memoria2000-2010-Castel\%C3\%A1.pdf

Tonucci, F. (1993). Aula de innovación educativa. Revista Aula de Innovación Educativa, 12, 147168. 


\section{Breve CV de los autores}

\section{Xosé Manuel Rosales}

Arquitecto por la Escuela Técnica Superior de Arquitectura de A Coruña y Máster en Proyectación Urbana y Territorial por la Universidad de A Coruña. Toda mi vida profesional está dedicada a la docencia, compatibilizada puntualmente con el ejercicio profesional como arquitecto. Soy Catedrático de Educación Secundaria y Profesor Asociado del Departamento de Proyectos Arquitectónicos y Urbanismo de la Universidad de A Coruña. Desde los inicios del Proxecto Terra en el año 2000 soy su coordinador al tiempo que coautor de algunas de las unidades didácticas y materiales audiovisuales elaborados. ORCID ID: 0000-0003-0229-0729. Email: proxectoterra@coag.es

\section{Marta Casal}

Licenciada en Sociología por la Universidad de A Coruña. Master en Género y Desarrollo por la Universidad Complutense de Madrid y Experta en Género y Políticas de Igualdad por la Universidad de A Coruña. Veinte años de experiencia profesional y activismo social en diferentes temáticas vinculadas a los derechos humanos, principalmente de las mujeres y las niñas. Ha trabajado para universidades españolas, organismos internacionales como la OIM y la OIT, ONG y administraciones públicas. Desde 2015 trabaja como técnica de Educación para el desarrollo en Arquitectura sin Fronteras Galicia en proyectos para promover el derecho al hábitat desde un enfoque interdisciplinar y de derechos. ORCID ID: 0000-0001-5964-5557. Email: casalmarta@hotmail.com

\section{Soledad Bugallo}

Arquitecta por la Escuela Técnica Superior de Arquitectura de A Coruña de la Universidad de A Coruña. Certificado de Adaptación Pedagógica. Diploma de Estudios Avanzados en Geografía Regional de Galicia. Máster en Arquitectura del Paisaje por la Universidad de Santiago de Compostela. Voluntaria de Arquitectura Sin Fronteras Galicia en diversos grupos de trabajo, Cooperación Internacional, Educación y Formación/Voluntariado. Desempeño de labores de coordinación de proyectos de gestión en al ámbito de la Educación para el Desarrollo y proyectos de Fortalecimiento Institucional. Formadora de curso en línea Hábitat a Escala Humana en aspectos de Hábitat y Género. Miembra del Comité Científico Congreso sobre Arquitectura y Cooperación al Desarrollo -Arcadia IV-. ORCID ID: 0000-0001-6658-0536. Email: solechou@hotmail.com

\section{Juan Antonio Ortiz}

Licenciado en Arquitectura Especialidad Urbanismo por la Escuela Técnica Superior de Arquitectura Universidad de A Coruña. Socio y voluntario desde 2010 en Arquitectura Sin Fronteras Galicia, en los Grupos de Trabajo de Educación y Comunicación, siendo responsable de este último desde 2015, y presidente de la Junta Directiva de la 
demarcación territorial Galicia desde 2016. ORCID ID: O000-0002-5525-2518. Email: juan.ortiz@icloud.com

\section{Lucía García-Cernuda}

Alumna de Proyecto Fin de Carrera de la Escuela Técnica Superior de Arquitectura de A Coruña. Técnica de gestión de ONG Arquitectura sen Fronteiras. Voluntaria de ONG Arquitectura sen Fronteiras en diversos grupos de trabajo, Cooperación Internacional y Educación. Estancias en Guatemala (4 meses) y apoyo en proyectos de diseño participativo. Coautora del Plan de Formación de Arquitectura sen Fronteiras. Responsable del grupo de Educación para el Desarrollo de Arquitectura sen Fronteiras. Desempeño de labores de coordinación de proyectos de gestión en al ámbito de la Educación para el Desarrollo y proyectos de Fortalecimiento Institucional. ORCID ID: ooo0-0002-6357-745 1. Email: luciacernuda@gmail.com 